\title{
Identification of non-equilibrium growth for Bitcoin Exchange rate: Mathematical derivation method in Islamic Financial Engineering
}

\author{
Nashirah Abu Bakar ${ }^{1}$, Sofian Rosbi ${ }^{2}$ \\ ${ }^{1}$ Islamic Business School, College of Business, Universiti Utara Malaysia \\ 06010 UUM Sintok, Kedah, Malaysia \\ ${ }^{2}$ School of Mechatronic Engineering, Universiti Malaysia Perlis, \\ 02600 Arau, Perlis, Malaysia
}

\begin{abstract}
:
Crptocurrency is a type of digital currency that using decentralized method for validating transaction. In the same time, the user of the Bitcoin is protected using hash algorithm. The value of Bitcoin is increased sharply in 2017 that creates much attention from investors. The objective of this study is to develop the mathematical model evaluate growth of Bitcoin exchange rate. The data selected in this study are starting from 1st January 2017 until 10th December 2017. This study validates the normality distribution of data using graphical and numerical method. Graphical method indicates the first difference of data distribution is a non-normal distribution. Then, Shapiro-Wilk statistical test confirmed that first difference of data distribution deviate from normal distribution. The non-normal distribution occurs because of the presence of outliers. Next, this study validates the mathematical modeling fordata trend using quadratic formula. Result indicates that the growth of Bitcoin exchange rate in moving towards non-equilibrium point. This concludes that the growth of the Bitcoin exchange rate is in unstable region. The contribution of this study is to help investors to understand the nature of Bitcoin exchange rate. This study performed analysis that helps investors to develop more efficient investment portfolio in gaining better profit and reducing loss.
\end{abstract}

\section{Keywords: Cryptocurrency, Bitcoin, Mathematical modelling, Equilibrium, Growth}

\section{Introduction}

The evolution of technology was produced many digital product such as digital currency known as bitcoin cryptocurrency. Bitcoin is a type of digital coins (cryptocurrency) which not issued by any government, bank or organization. Bitcoin is relying on cryptographic protocols and a distributed network of users to perform mining, storing, and transferring activities. Bitcoin is a decentralized electronic cash system initially designed and developed by Satoshi Nakamoto (Decker and Wattenhofer, 2013).

The price of the Bitcoin cryptocurrency has risen from December 2016, moving to USD 13282.29 in early December 2017. This value show the Bitcoin cryptocurrency was attracted more investors to involved in this transaction. There are many factors that influence the demand and supply for Bitcoin cryptocurrency. The main factor is Bitcoin cryptocurrency can generate high return. As investors, the main objective of the investment is to get high profit. Therefore, Bitcoin cryptocurrency are looking as a good platform for investment. The problem arise in Bitcoin cryptocurrency is either this cryptocurrency can fulfill the customer intention to receive high profit or not. This is because Bitcoin cryptocurrency transaction is involved with high speculation. As study by Abu Bakar and Rosbi (2017) the standard error of Bitcoin cryptocurrency is $4.458 \%$. This value is considered as high value of volatility. Therefore, high value of volatility indicates that the investment in Bitcoin is categorical as high risk investment.

Besides that, Bitcoin cryptocurrency involve with a complex process with the Bitcoin cryptocurrency in which encryption techniques are used to regulate the generation of units of currency (Abu Bakar, et al. 2017). Bitcoin cryptocurrency is types of currency that is non-physical, of which no banknotes and coin exist. Therefore, this study was conducted to fulfill the gap by developing the mathematical model evaluate growth of Bitcoin exchange rate. The data selected in this study are starting from 1st January 2017 until 10th 
December 2017. This study validates the normality distribution of data using graphical and numerical method.

\section{Literature Review}

Commerce on internet has come to rely almost exclusively on financial institutions serving as trusted third parties to process electronic payments (Nakamoto, 2009). The traditional method of financial transaction allows the customer to buy a product using a credit card to replace cash money (Awanis and Chi Cui, 2014). Credit cards are register and monitor under financial intermediaries. The use of credit cards has already become a convenient way to expand purchasing power (Braunsberger et al., 2004; Foscht et al., 2010; Teoh et al., 2013). Therefore this transaction is legal according to financial law. An electronic payment introduced that system based on cryptographic proof instead of trust, allowing any two willing parties to transact directly with each other without the need for a trusted third party (Kristoufek, 2013). Decentralized cryptocurrency is produced by the entire cryptocurrency system collectively, at a rate that is defined when the system is created and which is publicly known (Moore and Christin, 2013).

Although Bitcoin cryptocurrency transaction is not recognize by any financial intermediaries due to high risk, uncertainty process and involved with high volatility but the demand for Bitcoin cryptocurrency is increase. Abu Bakar and Rosbi (2017) observe the value of Bitcoin exchange rate from 1st January 2017 until 31st October 2017 (304 observations). The value of Bitcoin exchange rate on 1st January 2017 is 997.69, and the value of Bitcoin exchange rate on 31st October 2017 is 6142.46. There is increment of $515.7 \%$. This results show the high value of Bitcoin. Besides that, there are many opinions regarding Bitcoin cryptocurreny transaction. According to Simser (2015) Bitcoin is based on complex computer code supported by a robust community in a peer-to-peer network. Unlike other virtual currencies, Bitcoin appears to have obtained purchase and as such poses unique challenges to regulators.

Reynolds and Irwin (2017) Bitcoin cryptocurrency rely on a de-centralised system based on peer-topeer public key addresses, rather than having a central regulating body, such as a financial institution or bank, which reviews and monitors transactions. Therefore, this transaction allows potential criminal to be processed through cryptocurrencies, as the process of moving money is quicker and more efficient due to the bypassing of the regulatory controls that third-party institutions, such as banks, are legally bound to perform.

\section{Research Methodology}

This section develops statistical tests and mathematical modeling to observe the changes of bitcoin exchange rate. The research methodology starts with data selection, normality diagnostics procedure, outliers detection graphical method and mathematical modeling.

\subsection{Data selection}

This research selected Bitcoin exchange rate data starting form $1^{\text {st }}$ January 2017 until $10^{\text {th }}$ December 2017. Data are recorded from website of https://www.coindesk.com. Then, this study calculated first difference of Bitcoin exchange rate to evaluate the distribution of data.

\subsection{Normality}

This section describes the procedure to validate normal characteristics of the data distribution. The probability density of the normal distribution is:

$$
f(x)=\frac{1}{\sqrt{2 \pi \sigma^{2}}} e^{-\frac{(x-\mu)^{2}}{2 \sigma^{2}}}
$$

where:

$\mu$ is the mean or expectation of the distribution,

$\sigma$ is standard deviation, and

$\sigma^{2}$ is variance for data distribution.

Properties of a normal distribution: 
(a) The mean, mode and median are all equal.

(b) The curve is symmetric at the center. Data are distributed around the mean, $\mu$.

(c) Exactly half of the values are to the left of center and exactly half the values are to the right.

(d) The total area under the curve is 1 .

Next, this research using numerical test to validates the normality characteristics of data distribution, namely Shapiro-Wilk normality test. The null-hypothesis of Shapiro-Wilk normality test is that the population is normally distributed. Thus, if the p-value is less than the chosen alpha level, then the null hypothesis is rejected and there is evidence that the data tested are not from a normally distributed population. On the opposite side, if the p-value is greater than the chosen alpha level, then the null hypothesis that the data came from a normally distributed population cannot be rejected.

The Shapiro-Wilk test is a method to evaluate whether a random sample comes from a normal distribution. The test gives you a $\mathrm{W}$ value. The $\mathrm{W}$ value larger than chosen alpha $(0.05)$, will concludes the distribution of data follows normal distribution. The, if the data shows small values of $\mathrm{W}$, it is indicate your sample is not normally distributed. The formula for the $\mathrm{W}$ value is:

$W=\frac{\left(\sum_{i=1}^{n} a_{i} x_{(i)}\right)^{2}}{\sum_{i=1}^{n}\left(x_{i}-\bar{x}\right)^{2}}$

where:

$x_{i}$ is the value in the sample $\left(x_{1}, x_{2}, x_{3}, \ldots, x_{n}\right)$;

$x_{(i)}$ is the ordered sample values,$\left(x_{(1)}\right.$ is the smallest value in the sample);

$\bar{x}=\frac{\left(x_{1}+x_{2}+\ldots .+x_{n}\right)}{n}$ is the sample mean;

$a_{i}$ is constants that derived generated from the means, variances and covariances of the order statistics of a sample of size $n$ from a normal distribution. The calculation of $a_{i}$ is described in below equation.

$$
\left(a_{1}, a_{2}, a_{3}, \ldots, a_{n}\right)=\frac{m^{\mathrm{T}} V^{-1}}{\left(m^{\mathrm{T}} V^{-1} V^{-1} m\right)^{1 / 2}}
$$

where:

$V$ is the covariance matrix of those order statistics;

$m=\left(m_{1}, m_{2}, m_{3}, \ldots, m_{n}\right)^{\mathrm{T}}$

Element in Equation (4) is represented as:

$m_{1}, m_{2}, m_{3}, \ldots, m_{n}$ are the expected values of the order statistics of independent and identically distributed random variables sampled from the standard normal distribution

\subsection{Outliers detection method}

The box plot is a standardized way of displaying the distribution of data based on the five number summary: minimum (Lower extreme value limit), first quartile, median, third quartile, and maximum (upper extreme value limit). Figure 1 shows the box-plot diagram with outliers detection range (Abu Bakar and Rosbi, 2017).

There are five important elements in Box-and-whisker plots:

(i) Q1 - quartile 1, the median of the lower half of the data set 
(ii) Q2 - quartile 2, the median of the entire data set

(ii) Q3 - quartile 3, the median of the upper half of the data set

(iv) IQR - interquartile range, the difference from Q3 to Q1

(v) Extreme Values - the smallest and largest values in a data set

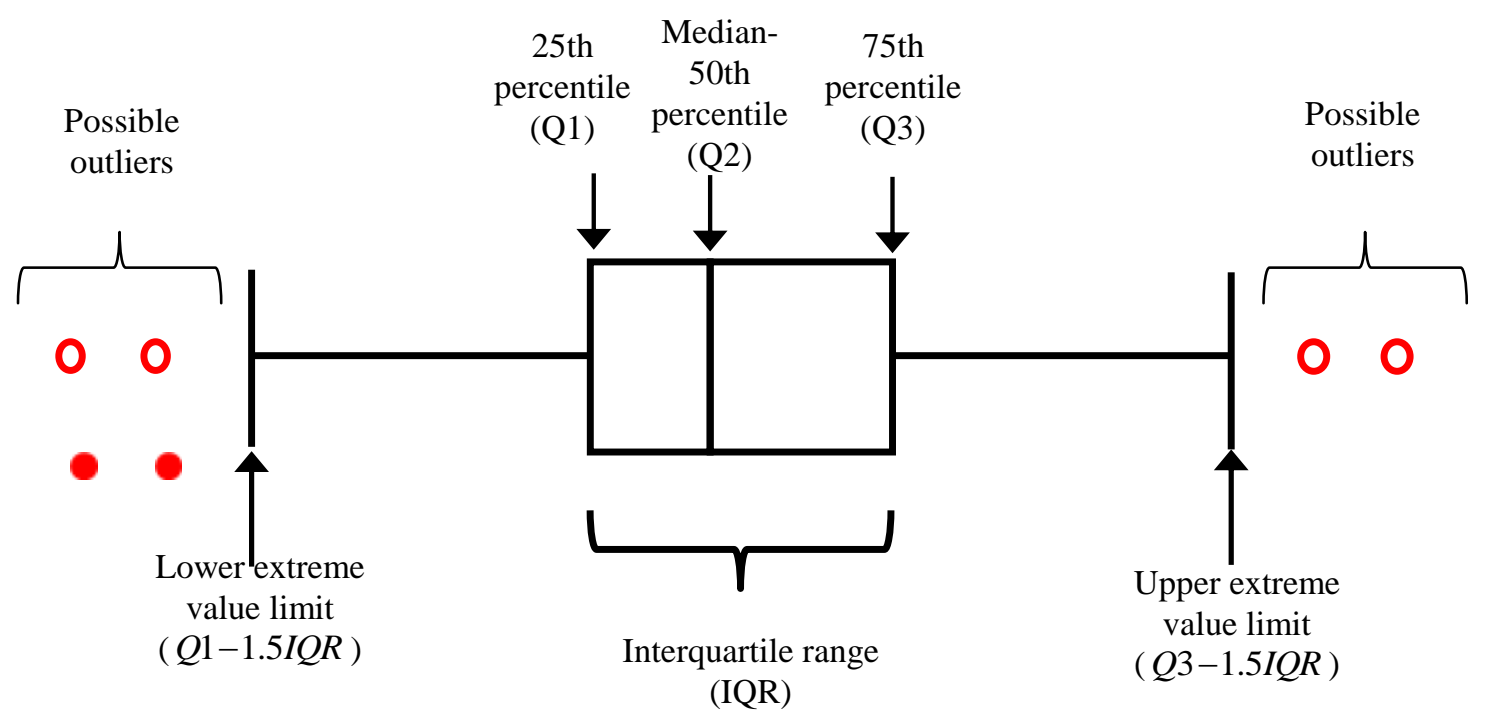

Figure 1: Box plot with outliers detection range

\subsection{Data trend using quadratic mathematical equation.}

This study implemented quadratic mathematical equation to represent the growth of exchange rate in first difference. Equation (5) describes the quadratic mathematical model.

$y=a x^{2}+b x+c$

Where,

$y$ is dependant variable;

$x$ is independent variable;

$\mathrm{a}, \mathrm{b}$ and $\mathrm{c}$ are constant.

\section{Result And Discussion}

\subsection{Data of Bitcoin exchange rate}

This objective of this study is to identify the mathematical equation for growth rate of Bitcoin exchange rate. Therefore, this study collected data of Bitcoin exchange rate from https://www.coindesk.com. The selected period are starting from $1^{\text {st }}$ January 2017 until $10^{\text {th }}$ December 2017 . The data for the each month is divided into three sections and recorded. There are 34 observations involved in this study. Figure 2 shows the dynamic movement of exchange rate for Bitcoin. The first observation in January 2017 shows value of exchange rate is USD 970.074 for every Bitcoin. The first observation in December 2017 indicates value of Bitcoin is USD 13282.29 for each of Bitcoin. The increment of Bitcoin exchange rate is 1269.2 percentage comparing value in December 2017 to initial value in January 2017. Figure 1 shows there is large increment in December 2017. 


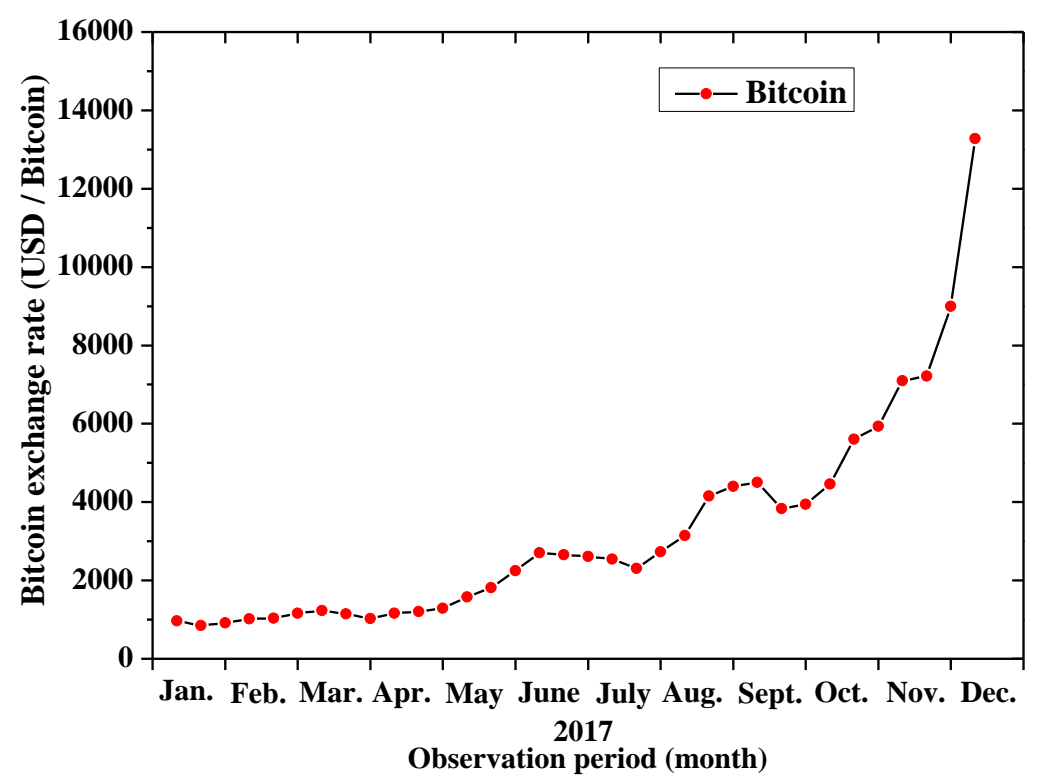

Figure 1: Movement of Bitcoin exchange rate

\subsection{First difference of Bitcoin exchange rate}

Then, this study performed first difference of Bitcoin exchange rate. The purpose of this step is to evaluate the rate of changes between observations. Figure 2 shows dynamic behavior of first difference for Bitcoin exchange rate.

The first difference of Bitcoin exchange rate calculated using below equation.

$\Delta$ ExRate $_{t}=$ ExRate $_{t}-$ ExRate $_{t-1}$

Where:

$\Delta$ ExRate $_{t}$ is first difference of Bitcoin exchange rate at observation period $t$;

ExRate $_{t}$ is Bitcoin exchange rate at observation period $t$, and

ExRate $_{t-1}$ is Bitcoin exchange rate at observation period $t-1$.

Figure 2 shows the value of Bitcoin exchange rate difference in January 2017 is USD (-124.615) for comparison first observation and second observation period. The minimum value of Bitcoin exchange rate difference is USD (-673.547) for second observation in September 2017. Meanwhile, the maximum value of Bitcoin exchange rate difference is USD 4282.196 for first observation in December 2017.

Next, this study validates the normality of Bitcoin exchange rate difference using graphical and numerical approach. Figure 2 shows the histogram for first difference of Bitcoin exchange rate. Figure 2 shows there is data that outside of normal distribution line. Therefore, data set of Bitcoin exchange rate difference is deviate from normal distribution.

Then, this study performed normal percentiles analysis. Figure 4 shows Normal pencentiles plot for first difference of Bitcoin exchange rate. Result shows the distribution of data is far from normal refernce line. Therefore, the distribution of data is a non-normal distribution. 


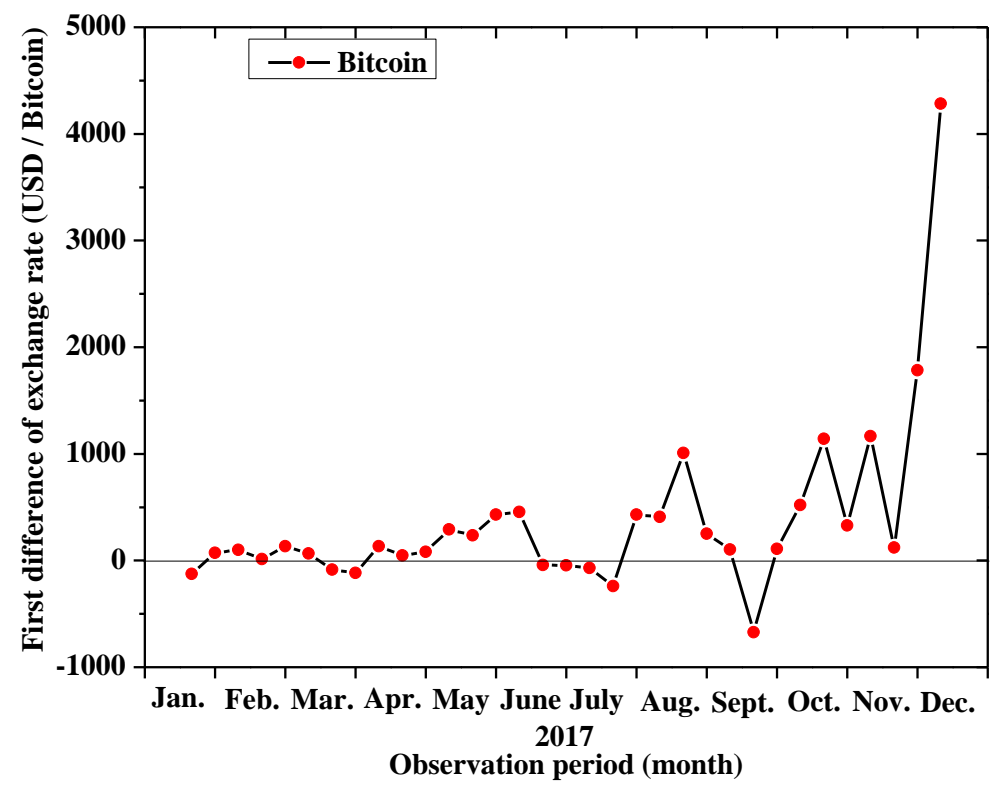

Figure 2: First difference of Bitcoin exchange rate

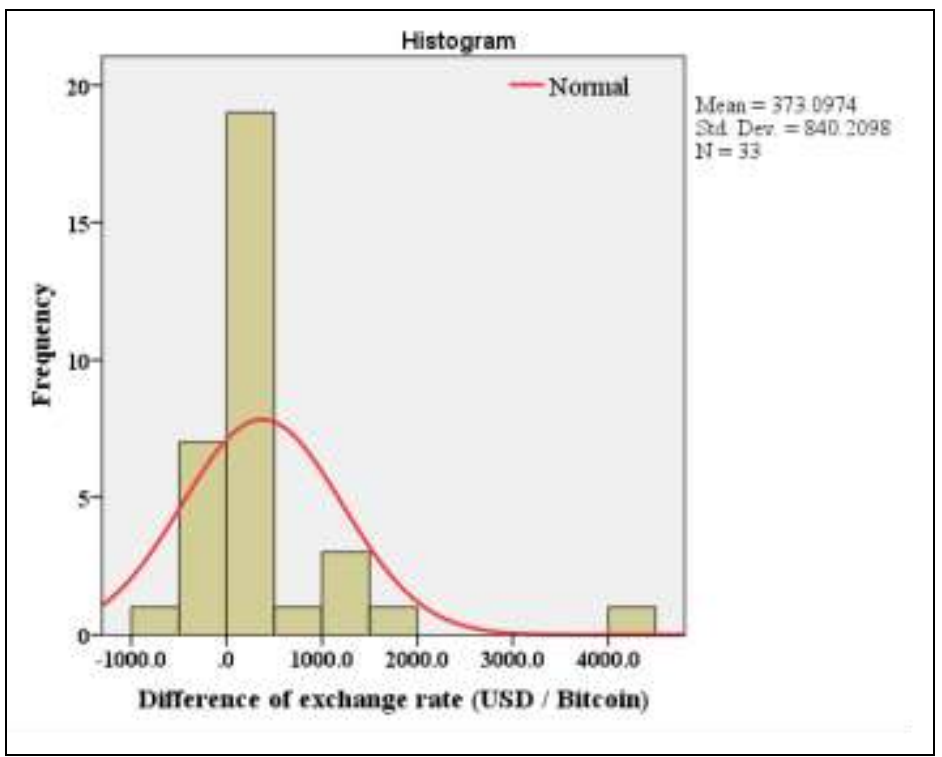

Figure 3: Histogram for first difference of Bitcoin exchange rate 


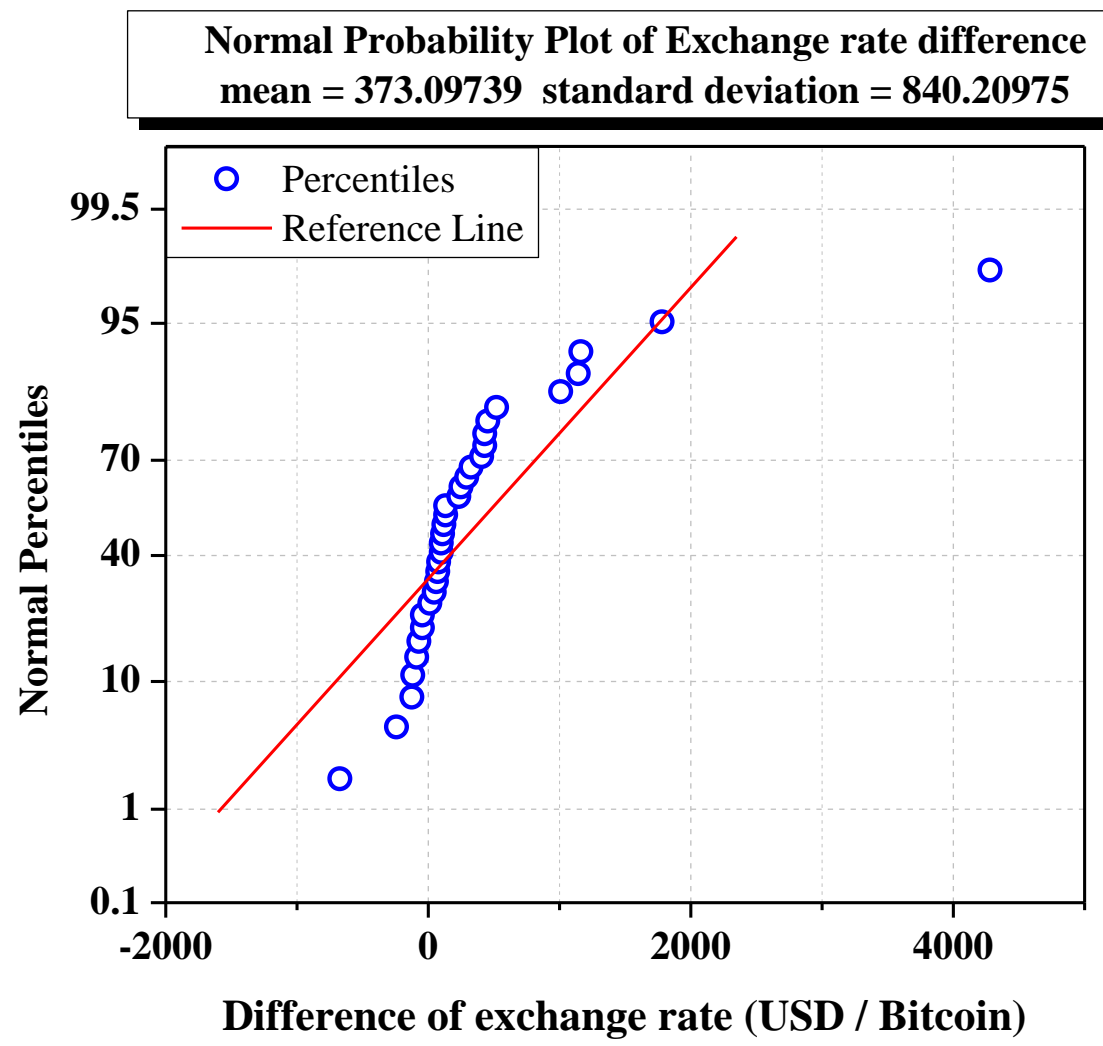

Figure 4: Normal pencentiles plot for first difference of Bitcoin exchange rate

Next, this performed numerical statistical test to validate the normality of the data distribution for Bitcoin exchange rate in first difference. This study selected Shapiro-Wilk normality test for the statistical normality checking. Table 1 shows the Shapiro-Wilk normality test for data of Bitcoin exchange rate in first difference. Table 1 indicates the significant value is 0.000 . The null hyphothesis for Shapiro-Wilk test is the data is follow normal distribution. The significant value concludes that data distribution rejected null hyphotesis. Therefore, data distribution of Bitcoin exchange rate in first difference is follows non-normal distribution.

Then, this study validate the reason of the non-normality characteristics for Bitcoin exchange rate in first difference.We performed Box-Whisker plot analysis to detect the outliers in the data. Figure 5 shows the Box-Whisker plot for first difference of Bitcoin exchange rate. Figure 5 shows there are two data points (red star mark) are outliers and 3 data points (blue circle mark) that are consider as possible outliers. The existance of these outliers contributes to the non-normalities of data.

Table 1 : Shapiro-Wilk statistical normality test

\begin{tabular}{|l|c|c|c|}
\hline & \multicolumn{3}{|c|}{ Shapiro-Wilk normality test } \\
\hline & Statistics & Degree of freedom & Significant value \\
\hline Difference of exchange rate & 0.628 & 33 & 0.000 \\
\hline
\end{tabular}




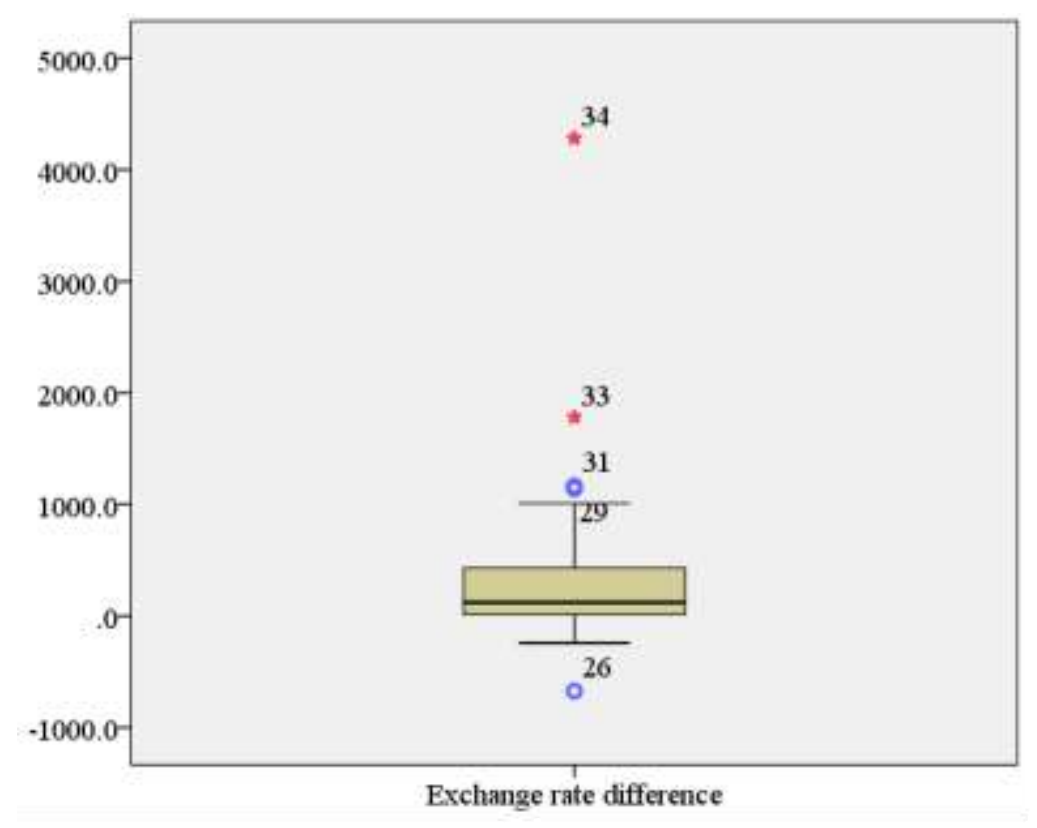

Figure 5: Box-Whisker plot

\subsection{Mathematical modelling for first difference of Bitcoin exchange rate using quadratic formula}

This section describes the growth of the Bitcoin exchange rate. Figure 6 shows data trend using polynomial function for first difference of Bitcoin exchange rate. Figure 6 shows the data trend can be represented by below equation.

$$
Y=4.16 x^{2}-105.65 x+551.43
$$

Where:

$Y$ is first difference of Bitcoin exchange rate $x$ is the order of observation

Figure 6 shows the value of exchange rate difference increase steadily and grow to a nonequilibrium point. Therefore, the growth of Bitcoin exchange rate difference is in stage of unstable region. Figure 7 shows residual of the difference between actual value and mathematical model. Result shows large residual exist in the $34^{\text {th }}$ observation. Therefore, the growth of Bitcoin exchange rate is moving towards nonequilibrium point.

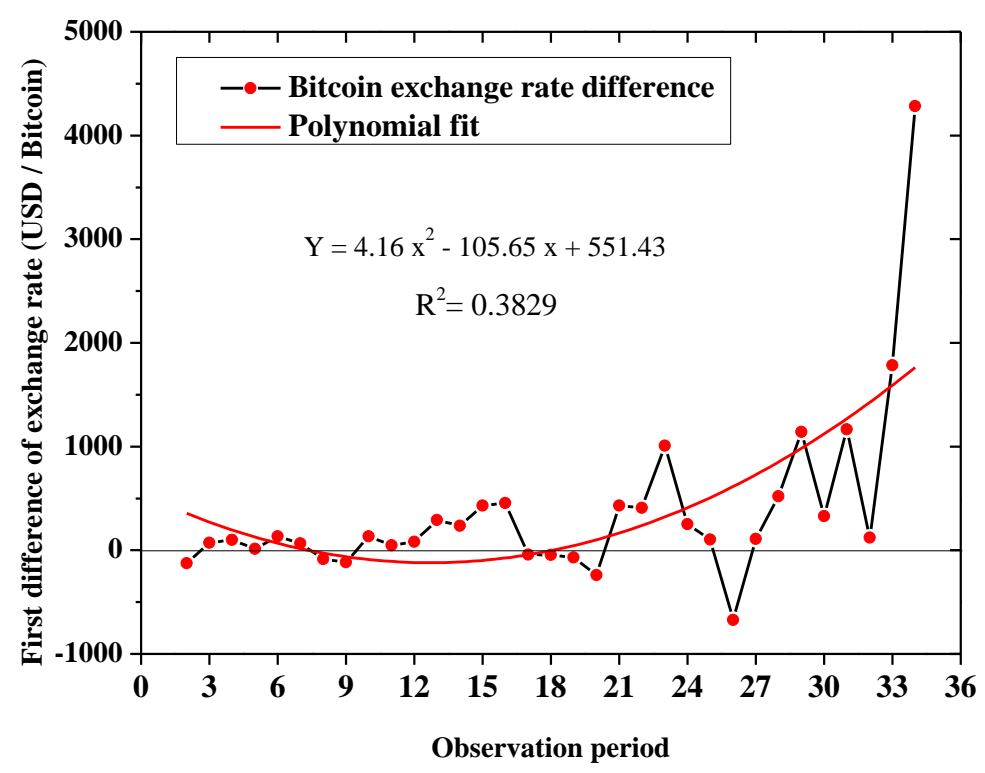

Figure 6: Data trend using polynomial function 


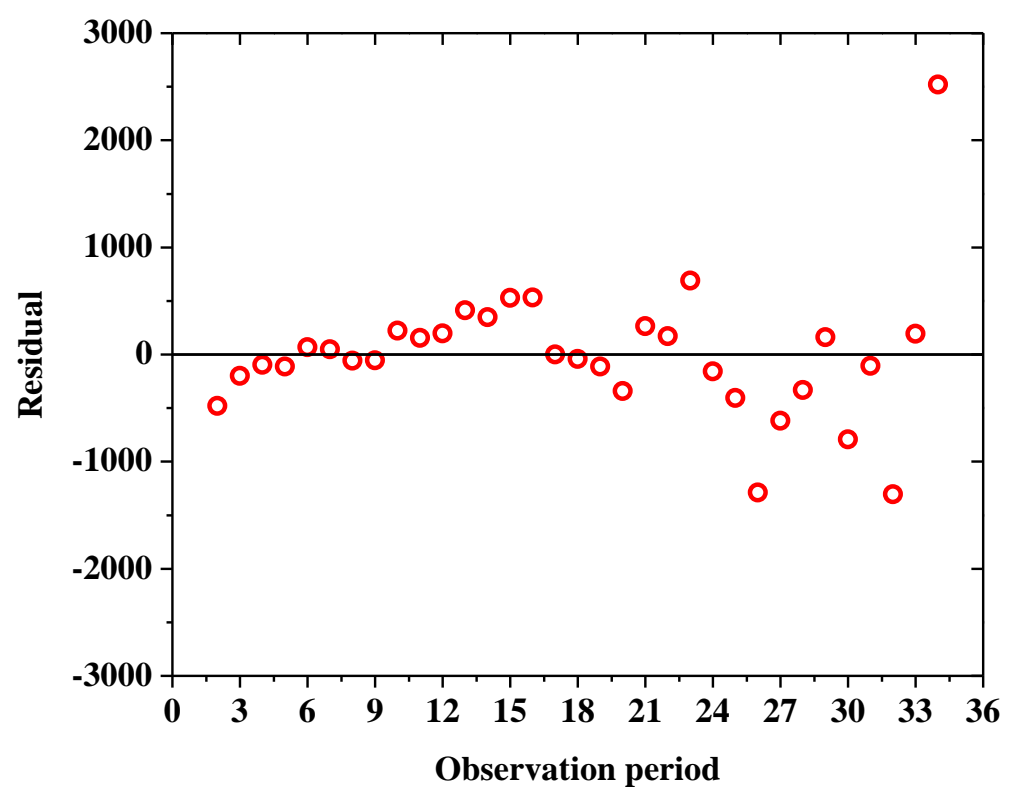

Figure 7: Residual for using polynomial function

\section{Conclusion}

The objective of this study is to develop mathematical modeling of Bitcoin exchange rate difference. The selected data in this study are starting from $1^{\text {st }}$ January 2017 until $10^{\text {th }}$ December 2017 . Then, the first differences of Bitcoin exchange rate are calculated. Next, the normality checking was performed to validate normality characteristics and outliers presence. Finally, mathematical modeling for growth of exchange rate difference was developed. Therefore, the main findings of this study are concluded as follow:

(i) The first observation in January 2017 shows value of exchange rate is USD 970.074 for every Bitcoin. The first observation in December 2017 indicates value of Bitcoin is USD 13282.29 for each of Bitcoin. The increment of Bitcoin exchange rate is 1269.2 percentage comparing value in December 2017 to initial value in January 2017.

(ii) The minimum value of Bitcoin exchange rate difference is USD (-673.547) for second observation in September 2017. Meanwhile, the maximum value of Bitcoin exchange rate difference is USD 4282.196 for first observation in December 2017.

(iii) This study selected Shapiro-Wilk normality test for the statistical normality checking. Result indicates the significant value is 0.000 . The null hyphothesis for Shapiro-Wilk test is the data is follow normal distribution. The significant value concludes that data distribution rejected null hyphotesis. Therefore, data distribution of Bitcoin exchange rate in first difference is follows non-normal distribution.

(iv) This study performed Box-Whisker plot analysis to detect the outliers in the data. Result shows there are two data points are outliers and 3 data points that are consider as possible outliers. The existance of these outliers contributes to the non-normalities of data.

(v) This study implemented data trend analysis using polynomial function for first difference of Bitcoin exchange rate. The data trend can be represented by below equation.

$$
Y=4.16 x^{2}-105.65 x+551.43
$$

Where:

$Y$ is first difference of Bitcoin exchange rate

$x$ is the order of observation

(vi) Next, this study using mathematical equation to model the growth of exchange rate difference. Results show the value of exchange rate difference increase steadily and grow to a nonequilibrium point.

The contribution of this study is to help investors to understand the nature of Bitcoin exchange rate. This study performed analysis that helps investors to develop more efficient investment portfolio in gaining better profit and reducing loss. 


\section{Future Research}

The further research of this topic can be extending to study the determinants that contribute to the dynamic behavior of Bitcoin exchange rate.

\section{References}

1. Abu Bakar, N. and Rosbi, S. (2017) High Volatility Detection Method Using Statistical Process Control for Cryptocurrency Exchange Rate: A Case Study of Bitcoin, The International Journal of Engineering and Science, Vol. 6(11), pp. 39-48

2. Abu Bakar, N. and Rosbi, S. (2017) Robust Statistical Normality Transformation method with Outlier Consideration in Bitcoin Exchange Rate Analysis, International Journal of Advances in Scientific Research and Engineering, Vol.3 (9), pp. 80-91

3. Abu Bakar, N., Rosbi, S. and Uzaki, K. (2017) Cryptocurrency Framework Diagnostics from Islamic Finance Perspective: A New Insight of Bitcoin System Transaction, International Journal of Management Science and Business Administration, Vol. 4 (1), pp.19-28

4. Awanis, S. and Chi Cui, C. (2014) Consumer susceptibility to credit card misuse and indebtedness, Asia Pacific Journal of Marketing and Logistics, Vol. 26 (3), pp.408-429

5. Braunsberger, K., Lucas, L.A. and Roach, D. (2004), The effectiveness of credit-card regulation for vulnerable consumers, Journal of Service Marketing, Vol. 18 (5), pp. 358-370

6. Decker,C. and Wattenhofer,R., Information propagation in the Bitcoin network, IEEE P2P 2013 Proceedings, Trento, 2013, pp. 1-10

7. Foscht, T., Maloles, C., Swoboda, B. and Chia, S.L. (2010), Debit and credit card usage and satisfaction: who uses which and why - evidence from Austria, International Journal of Bank Marketing, Vol. 28 (2), pp. 150-165

8. Kristoufek, L. (2013). BitCoin meets Google Trends and Wikipedia: Quantifying the relationship between phenomena of the Internet era. Scientific reports, 3, 3415.

9. Nakamoto, S. (2009) Bitcoin: A peer-to-peer electronic cash system", Retrieved from http://www.bitcoin.org /bitcoin.pdf

10. Moore, T., and Christin, N. (2013, April). Beware the middleman: Empirical analysis of Bitcoinexchange risk. International Conference on Financial Cryptography and Data Security (pp. 25-33). Springer, Berlin, Heidelberg.

11. Reynolds, P. and Irwin, A.S.M. (2017) Tracking digital footprints: anonymity within the bitcoin system, Journal of Money Laundering Control, Vol. 20 (2), pp.172-189

12. Simser, J. (2015) Bitcoin and modern alchemy: in code we trust, Journal of Financial Crime, Vol. 22(2), pp.156-169

13. Teoh, W.M.Y., Chong, S.C. and Yong, S.M. (2013) Exploring the factors influencing credit card spending behavior among Malaysians, International Journal of Bank Marketing, Vol. 31 (6), pp.481500 